\title{
Assessment of the Efficacy of Narrow-band UVB Treatment and Correlated Factors in Cutaneous Lichen Planus
}

\author{
(1) Tuğba Kevser Üstünbaș Uzunçakmak, (1) Defne Özkoca, (1) Özge Așkın, (1) Burhan Engin
}

Istanbul University Cerrahpasa-Cerrahpasa Faculty of Medicine, Department of Dermatology, Istanbul, Turkey

\section{ABSTRACT}

Background: Lichen planus (LP) is a papulosquamous skin disorder that affects less than $1 \%$ of the general population. As the cutaneous disease has a benign course, treatment depends on the location of the lesions; and the severity of the symptoms such as itching and hyperpigmentation. Narrow-band ultraviolet-B (nbUVB) has various cutaneous immunosuppressive effects, which makes it a potentially good treatment modality for cutaneous LP that is an immune-driven disease.

Materials and Methods: We present a total of 49 cutaneous LP patients treated with nbUVB three times weekly and have achieved complete remission. The age of the patient, the disease duration and the total number of sessions needed to achieve remission were noted. Spearman correlation coefficient was calculated.

Results: A total of 49 patients were included in this study. The mean age of the patients was 46 (9-71) years. The mean disease duration was 43.27 (1-300) months with a standard deviation of 75.4 months. The mean number of nbUVB sessions needed to achieve remission was 27.5 (12-63) sessions with a standard deviation of 9.6. No correlation between the number of sessions needed to achieve remission and the patient's age or disease duration were detected.

Conclusion: nbUVB treatment is a safe and effective treatment modality in cutaneous LP patients; and the number of sessions needed to achieve remission is independent of the patient's age and disease duration.

Keywords: Dermatology, Lichen planus, Phototherapy

\section{Introduction}

Lichen planus (LP) is a papulosquamous disorder, affecting less than $1 \%$ of the general population and mainly affects adult patients; however rare cases of pediatric LP have also been reported [1]. The mean age of diagnosis of cutaneous disease is 40 to 45 years of age; and 50 to 60 years of age for oral disease [2]. The disease has no gender predilection [1]. There are some reports of the disease having a female predilection, though. The disease has a self-limiting course; often resolving in a time period of a month to 7 years [3]. Infections, especially hepatitis C virus; environmental and genetic factors; and immune dysregulation are the possible etiologic factors for LP [1].
The clinical presentation of the disease varies according to the area involved. Mainly there are mucosal and cutaneous presentations. The cutaneous lesions are flat topped violaceus polygonal papules $[1,2]$. The papules may coalesce into large plaques. Both the papules and the plaques may have a white reticulation superimposed on them; which is referred to as the Wickham striae. Extremities are the most commonly affected areas; flexor wrists and ankles leading them all. The cutaneous lesions may have a zosteriform, blaschkoid or inverse distribution as well. The patients may complain of intense pruritus, which leads to secondary excoriations [3]. The oral disease has a more chronic course; it presents with a lace-like white 
reticulation most commonly symmetrically located on the buccal mucosa. Erosions and ulcerations may occur [2]. The disease may involve the hair follicles, as lichen pilanopilaris, and the nail unit as well [4].

As the cutaneous disease has a benign course, treatment depends on the location of the lesions; and the severity of the symptoms such as itching and hyperpigmentation. The mucosal disease, instead, has a more chronic course and should be treated due to the risk of squamous cell cancer arising within it. The first line treatment modality for cutaneous lesions is high potency topical corticosteroids [2]. Other treatment modalities used for cutaneous LP, especially for more wide-spread disease, are systemic corticosteroids, acitretine, sulfasalazine, griseofulvin, hydroxychloroquine and narrow-band ultraviolet-B (nbUVB) $[5,6]$. The treatment of mucosal lesions is beyond the scope of this article.

nbUVB is a phototherapy modality in which a light of 310 to 315 $\mathrm{nm}$ wavelength is emitted from a light source; the most commonly used wavelength being $312 \mathrm{~nm}$. The nbUVB has inhibitory effects on the Langerhans cells along with inducing anti-inflammatory effects via Intracellular Adhesion Molecule 1 suppression. nbUVB is unique for its selective wavelength and fewer side effects; and it can even be used safely in children and in pregnant women [7]. nbUVB has been proven to be efficacious in the treatment of cutaneous LP. The previously used treatment protocols had an average energy of $9-17 \mathrm{~J} / \mathrm{cm}^{2}$ and nbUVB was applied three to four times weekly $[7,8,9,10,11]$. The total number of treatment sessions needed was found to be independent of age and disease duration $[9,11]$. This study aims at determining the correlation between the patient's age and the total number of treatment sessions needed to achieve remission; and between the disease duration and the total number of treatment sessions needed to achieve remission, in a larger study group.

\section{Patients and Methods}

This is a retrospective study evaluating the relationship between total number of nbUVB treatment sessions needed to achieve remission, with the disease duration and the age of the patient, in cutaneous LP patients. The patient files of the cutaneous LP patients who received nbUVB treatment at the Phototherapy Outpatient Unit of our department between the January 2018 and January 2020, were analyzed. Only the patients who have achieved complete remission were included in this study: a total of 49 patients. The following parameters were noted from the files: age at the time of treatment (in years), the disease duration (in months) and the total number of sessions needed to achieve remission. The nbUVB treatment protocol for all patients was as follows: a starting dose of 0.30 J with a $15 \%$ dose increment every 2 sessions, three sessions per week. The patients wore protective gear for the ocular and genital areas. The nbUVB device that was used is Waldmann W- UV 7002. Topical mouisturizers and topical medium-potency corticosteroids were offered to the patients. Relapse and recurrence rates were not analyzed in this study. The approval of Istanbul University Cerrahpasa-Cerrahpasa Faculty of Medicine Ethics Committee was obtained before the study was initiated. Patient consent was taken from all patients before the phototherapy.

\section{Statistical Analysis}

SPSS was used for statistical analysis. Non-parametric tests were performed since the data did not show a normal distribution. spearman correlation coefficient was calculated.

\section{Results}

A total of 49 patients were included in this study. The mean age of the patients was 46 years with a standard deviation of 13.6; the oldest patient was 71 years old and the youngest patient was 9 years old. The mean disease duration was 43.27 months with a standard deviation of 75.4 months; the shortest disease duration was 1 month and the longest was 300 months. The mean number of nbUVB sessions needed to achieve remission was 27.5 sessions with a standard deviation of 9.6; the minimum number of sessions was 12 and the maximum was 63. The data did not show a normal distribution pattern. The descriptives of the data is summarized in Table 1.

The Spearman correlation coefficient between the age of the patient and the number of sessions to achieve remission was calculated as -0.158 . The Spearman correlation coefficient between the disease duration and the number of sessions needed to achieve remission was -0.270 . Both of the relationships were statistically insignificant. Thus, there is no correlation between the number of sessions needed to achieve remission and the patient's age or disease duration.

\section{Table 1. Descriptives of the data}

\begin{tabular}{|l|l|l|l|l|}
\hline & Mean & Standard deviation & Minimum & Maximum \\
\hline Age (years) & 46 & 13.6 & 71 & 9 \\
\hline Disease duration (months) & 43.37 & 75.4 & 1 & 300 \\
\hline Number of sessions needed to achieve remission & 27.5 & 9.6 & 12 & 63 \\
\hline
\end{tabular}




\section{Discussion}

Epidermal cell destruction orchestrated by T-lymphocytes is the underlying pathogenetic mechanism for LP [10]. nbUVB has various cutaneous immunosuppressive effects, which makes it a potentially good treatment modality for cutaneous LP that is an immunedriven disease. nbUVB decreases the number of Langerhans cells in the epidermis; increases the production of anti-inflammatory cytokines, prostanoids and neuropeptides from the keratinocytes: interleukin-10, alpha-melanocyte stimulating hormone and prostaglandin E2; increases the expression of interleukin-1 receptor-2; suppresses the neurophils and decreases Intracellular Adhesion Molecule 1 [12].

Taneja and Taylor [10] were the first to report the efficacy of nbUVB treatment in cutaneous LP patients. A year later, Saricaoğlu et al. [8] studied the efficacy of nbUVB in 10 cutaneous LP patients. In their study, $50 \%$ of the patients achieved remission at the end of the $30^{\text {th }}$ treatment session; and $80 \%$ achieved remission by the end of the study. They also concluded that nbUVB was effective in the treatment of cutaneous LP; and that nbUVB was safer than psoralen-UVA treatment and the cumulative UV dose was lower in nbUVB.

Iraji et al. [7] compared the efficacy of nbUVB in disseminated cutaneous LP to systemic corticosteroids. A total of 46 patients, who had at least $20 \%$ body surface area involvement and had pruritus resistant to oral anti-histaminic drugs, were divided into two groups: the first group received nbUVB three times weekly for 6 weeks at a maximum dose of $9 \mathrm{~J} / \mathrm{cm}^{2}$; and the second group received oral prednisolone $0.3 \mathrm{mg} / \mathrm{kg} /$ day for 6 weeks. nbUVB was found to be more effective than systemic corticosteroids in the treatment of disseminated cutaneous LP. Furthermore, it serves a good treatment alternative in patients for whom systemic corticosteroids or other immunosuppressive drugs are contraindicated.

There are two previous studies in the literature which have studied the parameters that might affect the treatment response of $L P$ to
nbUVB. Habib et al. [11] conducted a study on twenty patients. They found that the average number of sessions required for remission was 30; which is close to our result of 27.5. They also concluded that the required session number was independent of patient's gender, skin phototype, disease duration and patient's age. Pavlotsky et al. [9] investigated the effect of gender, age, phototype, disease duration, previously used treatment modalities and comorbid diseases on the treatment response and the cumulative exposure dose in 43 cutaneous LP patients who were treated with nbUVB. They concluded that the complete response rate and the cumulative exposure dose, thus the total number of treatment sessions required to achieve remission, were independent of all the parameters that were mentioned above. Similar to Habib et al. [11] and Pavlotsky et al. [9], we also report that the total number of treatment sessions required to achieve remission is independent of the patient's age and the disease duration. Moreover, our sample size (49) was greater than Habib et al. [11] and Pavlotsky et al. [9] (Table 2).

\section{Conclusion}

nbUVB treatment is a safe and effective treatment modality in cutaneous LP patients. This study evaluated the effect of patient's age and disease duration on the total number of treatment sessions needed for complete remission. The number of sessions needed is independent of the patient's age and disease duration.

\section{Ethics}

Ethics Committee Approval: The approval of Istanbul University Cerrahpasa-Cerrahpasa Faculty of Medicine Ethics Committee was obtained before the study was initiated (decision no: A-05, date: 27.05.2020).

Informed Consent: Patient consent was taken from all patients before the phototherapy.

Peer-review: Internally peer-reviewed.

Table 2. Efficacy of narrow-band UVB in lichen planus

\begin{tabular}{|l|l|l|l|l|}
\hline Authors & Year & $\begin{array}{l}\text { Number of } \\
\text { patients }\end{array}$ & $\begin{array}{l}\text { Maximum dose } \\
\left(\mathbf{J} / \mathbf{c m}^{2}\right)\end{array}$ & Conclusions \\
\hline Taneja and Taylor [10] & 2002 & 5 & Not mentioned & nbUVB is a treatment alternative for generalized cutaneous LP \\
\hline Saricaoğlu et al. [8] & 2003 & 10 & 17 & Not mentioned \\
\hline Habib et al. [11] & 2005 & 20 & $\begin{array}{l}\text { nbUVB is a treatment alternative for generalized cutaneous LP } \\
\text { nkin phototype, gender, age and disease duration do not influence the } \\
\text { treatment response }\end{array}$ \\
\hline Pavlotsky et al. [9] & 2007 & 43 & 11 & $\begin{array}{l}\text { The treatment response of generalized cutaneous LP is independent } \\
\text { of gender, age, skin phototype, disease duration, previous treatment } \\
\text { modalities and comorbid diseases }\end{array}$ \\
\hline Iraji et al. [7] & 2011 & 46 & 9 & $\begin{array}{l}\text { nbUVB is more efficacious than systemic corticosteroids in the treatment } \\
\text { of generalized cutaneous LP (p=0.008) }\end{array}$ \\
\hline
\end{tabular}




\section{Authorship Contributions}

Surgical and Medical Practices: T.K.Ü.U., D.Ö., Ö.A., B.E., Concept: T.K.Ü.U., Ö.A., Design: T.K.Ü.U., Ö.A., B.E., Data Collection or Processing: T.K.Ü.U., D.Ö., Ö.A., B.E., Analysis or Interpretation: T.K.Ü.U., Ö.A., B.E., Literature Search: T.K.Ü.U., D.Ö., Writing: T.K.Ü.U., D.Ö.

Conflict of Interest: No conflict of interest was declared by the authors.

Financial Disclosure: The authors declared that this study received no financial support.

\section{References}

1. Tziotzios C, Lee JYV, Brier T, Saito R, Hsu CK, Bhargava K, Stefanato CM, Fenton DA, McGrath JA. Lichen planus and lichenoid dermatoses: Clinical overview and molecular basis. Acad Dermatol 2018;79:789-804.

2. Cleach LL, Olivier Chosidow O. Clinical Practice. Lichen Planus. N Engl J Med 2012;366:723-732.

3. Lehman JS, Tollefson MM, Gibson LE. Lichen Planus. Int J Dermatol 2009:48:682-694.
4. Berger TG. Lichen Planus. JAMA Dermatol 2015;151:356.

5. Fazel N. Cutaneous Lichen Planus: A Systematic Review of Treatments. J Dermatolog Treat 2015;26:280-283.

6. Atzmony L, Reiter O, Hodak E, Gdalevich M, Mimouni D. Treatments for Cutaneous Lichen Planus: A Systematic Review and Meta-Analysis. Am J Clin Dermatol 2016;17:11-22.

7. Iraji F, Faghihi G, Asilian A, Siadat AH, Larijani FT, Akbari M. Comparison of the Narrow Band UVB Versus Systemic Corticosteroids in the Treatment of Lichen Planus: A Randomized Clinical Trial. J Res Med Sci 2011;16:1578-1582.

8. Saricaoğlu H, Karadogan SK, Başkan EB, Tunali ș. Narrowband UVB Therapy in the Treatment of Lichen Planus. Photodermatol Photoimmunol Photomed 2003;19:265-267.

9. Pavlotsky F, Nathansohn N, Kriger G, Shpiro D, Trau H. Ultraviolet-B Treatment for Cutaneous Lichen Planus: Our Experience With 50 Patients. Photodermatol Photoimmunol Photomed 2008;24:83-86.

10. Taneja A, Taylor CR. Narrow-band UVB for Lichen Planus Treatment. Int J Dermatol 2002;41:282-283.

11. Habib F, Stoebner PE, Picot E, Peyron JL, Meynadier J, Meunier L. Narrow Band UVB Phototherapy in the Treatment of Widespread Lichen Planus. Ann Dermatol Venereol 2005;132:17-20.

12. el-Ghorr AA, Norval M. Biological effects of narrow-band (311 nm TL01) UVB irradiation: A review. J Photochem Photobiol B 1997;38:99-106. 